Schweiz. Z. Path. Bakt. 1939;2:I-IV

\title{
Contents, Vol. 2, 1939
}

Schweizerische Zeitschrift

für

\section{ALLGEMEINE PATHOLOGIE UND BAKTERIOLOGIE}

Revue Suisse de Pathologie générale et de Bactériologie

Unter ständiger Mitarbeit zahlreicher Fachgelehrter herausgegeben von

A. v. Albertini - A. Grumbach - H. Mooser Zurich

Vol. II

S.iא

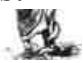

1939

BASEL (Schweiz) S. K A R G E R NEW YORK

Holbeinat $\operatorname{cas} \beta \mathrm{e} 22 \quad 215$ Fourth Avenue

For USA.: Nordeman Publishing, Inc., Dept. S. Karger, 215 Forth Avenue, New York, N.Y.

Alle Rechte vorbehalten

Printed in Switzerland Druck von Friedrich Reinhardt AG. iu Basel

INDEX.

Askanazy, M., Quand les vaisseaux ombilicaux (placen-

taires) entrent-ils dans la tête du foetus ? ... 1

Beeuwkes, H., Isolierung des Virus von Herpes febrilis in

einem Falle von Herpes Zoster thoracalis .... 334

Braun, H., Ueber das aerobe und anaerobe Wachstum der

Bakterien unter der Einwirkung von Kaliumzyanid.

4. Mitteilung (Staphylokokken, Strepto-, Entero- und

Pneumokokken) 309

- und E. Caspari, Kann Culex pipiens bakterielle Infek-

tionserreger verbreiten? 175

Brunner, W., Ueber abszedierende Brucella abortus Bang-

Strumitis $\quad 100$

Brzezinski, S., Untersuchungen über die normalen Anti-

staphylolysine des menschlichen Serums .... IS

Caspari, E., vide Braun, $\mathrm{H}$.

Collier, W. A., Untersuchungen über Ratten-Polyarthritis.

Versuche zum Nachweis neutralisierender Antistoffe 65 Collier, W. A., vide Mochtar, A.

Eugster, J., Methode zur Untersuchung der biologischen

Wirkung der Ultrastrahlung (K. S.) 109

Francillon, M. R., Contribution à Гétude de Гanatomie mi- 
croscopique de la malformation luxante de la hanche 77 Friedlí, A., Zur Pathogenese der diffusen Hämangiomatosis

der Leber $\quad 353$

Geiser, P., Tod im Asthmaanfall bei einem zehnjährigen

Knaben $\quad 373$

Grumbach, A., und H. W. Hotz, Bacillus anaerobius di-

phtheroides als Erreger einer Septikopyämie . . . 230 Hoff, H., und J. Shabí, Die intrauterine

Uebertragung der

Enzephalitis 263

Hotz, H. W., vide Grumbach, A.

Jaffé, R., Pathologisch-anatomische Untersuchungen über

das strikturierende Rektumgeschwür (Lymphogranu-

lomatosis inguinalis oder Nicolas-Favresche Krankheit) 31

Kaiser, P., Ueber die Beteiligung des Histamins an der Ent-

stehung von Trans- und Exsudaten 289

Meyenburg, H. v., Zur pathologïschen Anatomie und Pa-

thogenese der Ileitis regionalis, bzw. terminalis 217

Mochtar, A., und W. A, Collier, Die Ratten als Virusreser-

voir bei Leptospirose 319

Munzinger, W., Ergebnisse der Pneumokokkenagglutina-

tion im Patientenserum 389

Scheidegger, S., Heterotope Knochenbildung. (Beitrag zur

Frage der Metaplasie) 153

Shabi, 3., vide Ho ff, H.

Storck, H., Tierexperimentelle Untersuchungen über den

Einfluß von Vitamin C auf allergische Vorgänge . . 338

Wiederkehr, W., Zur Epidemiologie der Aktinomykose

Will, G., Zur Pathogenese der Lungen-Enchondrome .

Wohlwill, F., Ueber Hepatitis interstitialis infiltrativa dif-

fusa 240

UEBERSICHTSREFERATE - REVUES

Kaiser, P., Ueber die Beteiligung des Histamins an ent-

zündlichen Prozessen 267

Kallós, P., Neuere Entwicklung der Bakteriologie und Im-

munbiologie der Tuberkulose 47

Lépine, P., Sur Tétiologie des Méningites lymphocytaires

primitives 204

Nabholz, H., Periarteriitis nodosa generalisata Kußmaul-

Maier 112

VARIA 6. Internationaler Zellforscherkongreß 1940 in Stockholm 144 